\title{
SURGICAL APPROACH ON MALIGNANT TUMOR OF THE ORAL CAVITY AND PHARYNX
}

\author{
S.V.G. Bertesteanu ${ }^{1}$, B. Popescu ${ }^{1}$, D. Ionescu ${ }^{2}$, C.R. Popescu ${ }^{1}$, T. Ionescu ${ }^{3}$, D. Mirea ${ }^{4}$, R. Grigore ${ }^{1}$ \\ ${ }^{\text {I}}$ E.N.T. Department, Coltea Clinical Hospital, Bucharest, Romania \\ ${ }^{2}$ E.N.T Department, ,, Victor Gomoiu “ Clinical Children Hospital, Bucharest, Romania \\ ${ }^{3}$, ,Titu Maiorescu“ University, Faculty of Dental Medicine, Bucharest, Romania \\ ${ }^{4}$ E.N.T. Department, Elias Emergency Clinical Hospital, Bucharest
}

\begin{abstract}
Background. Malignant tumors of the head and neck have an increasing incidence in the population worldwide, including population of Romania. From all the tumors of the head and neck region oral cavity and pharynx tumors, with extension towards the larynx, have a great impact on the overall survival rates, functional implications and quality of life of the patients. Despite early symptoms, patients have a tendency to present themselves to the specialist in late stages of the disease because of ignorance, fear of diseases or bad advice from unknowing persons. This situation is a great challange for the oncology team considering the poor prognosis of locally advanced cancer. Development of malignant tumors of the oral cavity and pharynx in specific anatomical regions leads to particular ways of approach for each tumor located in different regions of the head and neck. Surgery is the main thod in locally advaced tumors of the oral cavity and pharynx alongside with neck dissection for regional metastasis in lymph nodes.

Material and methods. Tumor resection needs to be performed with oncological safe margins of resection which means that a good exposure of the site of the tumor needs to be obtained. In the case of oral cavity and pharynx tumors there are several possibilities of approach. T1 and T2 tumors can be resected by using a transoral approach. T3 tumors have a great local extension thus the need for a better exposure of the site of location through the check. The greater tumors in diameter, T4 tumors, need to be approached by a transmandibular procedure so that the entire tumors can be resected with oncological safe margins of resections. The authors present their experience with the locally advanced malignant tumors of the oral cavity and pharynx in Coltea E.N.T. Clinic.

Results. The use of the transmandibular approach in managing cases of locally advanced tumors of the oral cavity and pharynx has some complications that can be avoided if a proper surgical technique is used. Patients operated using the transmandibular technique have a good quality of life despite some complications associated with radiotherapy performed after surgery.
\end{abstract}

Keywords: cancer, transmandibular approach, oral cavity, pharynx

\section{INTRODUCTION}

Malignant tumors of the head and neck have an increasing incidence in the population worldwide, including population of Romania. From all the tumors of the head and neck region oral cavity and pharynx tumors, with extension towards the larynx, have a great impact on the overall survival rates, functional implications and quality of life of the patients. Despite early symptoms, patients have a tendency to present themselves to the specialist in late stages of the disease because of ignorance, fear of diseases or bad advice from unknowing persons. This situation is a great challange for the oncology team considering the poor prognosis of locally advanced cancer. Development of malignant tumors of the oral cavity and pharynx in specific anatomical regions leads to particular ways of approach for each tumor located in different regions of the head and neck. Surgery is the main thod in locally advaced tumors of the oral cavity and pharynx alongside with neck dissection for regional metastasis in lymph nodes.

Malignant tumors located in the oral cavity and pharynx present early symptoms that bring the patient to the physician in early stages of disease. Disphagia, odinopahgia, reflex ear pain, impairment of speach, hipersalivation are some of the most im-

Corresponding author:

Bogdan Popescu, M.D., PhD, Coltea Clinical Hospital, Bucharest, I.C. Brătianu Ave. no. 1-3, 3rd District, Bucharest, Romania E-mail: dr.bpopescu@gmail.com 
portant symtoms that appear in the case of such malignant tumors. The first step of therapy approach is the biopsy in order to evaluate the nature of the tumor. After establishing the correct diagnosis patients chose between different ways of treatment for cancer. The locally advanced cancer benefits from surgical therapy which implies the use of mandibulotomy, median or lateral, for an optimal exposure of the tumor site, oral cavity, pharynx, parapharynx space. (1) The parapharingeal space is a particular anatomic region to be approached and the surgical technique requires the disection of the base of the skull and visualisation through the nasopharynx. (2)

One of the most important oncological principal that needs to be taken into consideration when operating on patients with head and neck cancer is to remove the tumor along with the lymph nodes that drain the lymph from the tumor site. This means that a surgeon needs to be extremely well trained and this type of surgery needs to be performed in specailised oncology facilities. Neck dissection is of outmost importance due to the fact that lymph node involvement drops the survival rates with $50 \%$ in all stages of disease.

The history of the procedure comes from a long way back. The first mentioning of the technique belongs to Roux in 1836 , followed by Trotter. $(3,4)$ The sectioning of the mandible can be performed by using either cold instruments, cisel, Gigli saw (1894), or by mechanical power drive instruments introduced by McGregor. (4,5) The advance in power instruments and materials for osteosynthesis lead to imporved prognosis, less complications, improved function and a better quality of life.

There are two types of osteotomies that can be performed, lateral or median, depending on the localisation of the tumor. The main landmark for mandibulotomy is the mental foramen. The blood vessels traveling through the foramen need to be preserved if the resection allows it in order to ensure a better blood flow inside the bone. The blood flow is essential for reducing the possibility of radionecrosis after radiotherapy. In our clinic some $65 \%$ of the interventions including mandibulotomy approach of the oral cavity and pharynx malignant tumors are median.

\section{MATERIALS AND METHOD}

One study performed in our clinic on a period of 3 years included 119 patients that underwent surgical therapy with mandibulotomy for cancer of the oral cavity and pharynx.
The diagnosis is very important for these patients so we need to follow a diagnosis protocole that includes clinical examination, imaging studies, CT scans, MRI and plain X-ray investigations, panendoscopy of all the regions of the oral cavity, pharynx and larynx. We use the TNM system for staging the tumor and the surgical indication is established after examining all the data about the tumor. Due to the extent of the malignant process in late stage disease there is a need for interdisciplinary examinations including neurological, stomatological, cardiological, intensive care and anesthesiology examination, nutritional status and general surgery examination to establish the opportunity of an alternative way to feed the patient. The main aspect of the case management is the oncology decision made by the oncology board. The therapy plan is established after assessing all the data from the diagnosis protocole. The surgical treatment leads to cervical and facial scars this meaning that the patient needs to be very well informed about the functional and aesthetic impairment. After being presented with all the therapy options, complications, benefits, incidents and accidents the patient needs to sign the informed consent form. This consent form needs to be exhaustive and to include all the information given to the patient in an oral, written or media form. The best way to obtain the constent from the patient is to record the entire disscussion with the patient. At the end of the disscussion the patient needs to confirm that he/she understood al the data presented.

The technique for performing mandibulotomy differs from median to lateral. Medial mandibulotomy can be divided into midline which is performed between the two central incisors and paramidline. The later is performed in between the incisor and the canine teeth.

Teeth need to be examined and if possible the canine teeth will be preserved due to the higher sheer force they can outstand suitable for tearing food. The canine teeth have a better functionality than the incisors. However, keeping one or the other is strictly related to te type of mandibulectomy needed. Stomatological examination can also assess the integrity of the roots. We must not preserve teeth with bad roots for this is an inflamation site.

Muscles of the floor of the mouth and the tongue need to be visualised by the imaging studies on the internal surface of the mandible. The body of the mandible needs to be investigated so that we can establish if there is a need for a partial mandibulectomy. There are imaging criteria that need to be observed. The entire assessment of the mandible if 
critical for the surgical technique. The midline mandibulotomy does not preserve the insertion of the genioglossus and the geniohyoid muscles this leading to impairment in movement of the tongue and swallowing. (7) The functional recovery is better when using the lateral mandibulotomy. Still, a surgeon must not chose functionality over oncological outcome.
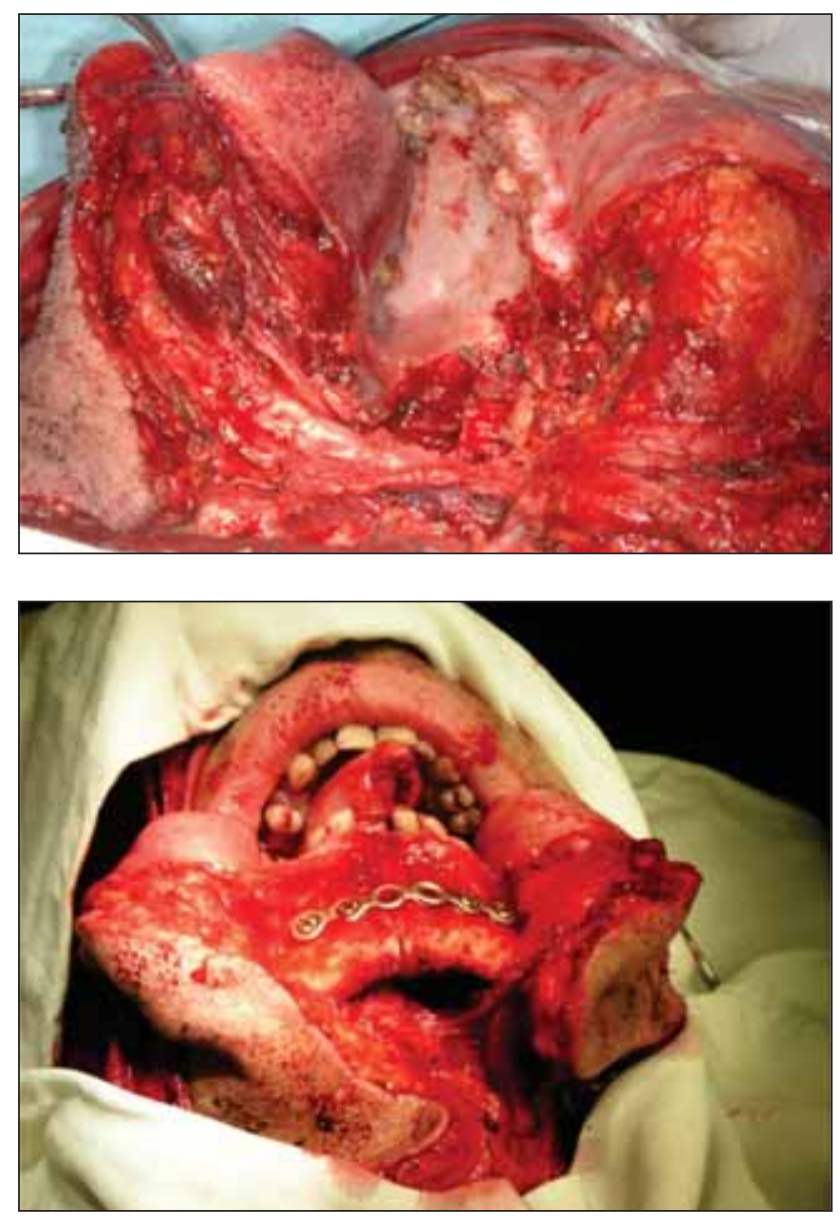

FIGURE 1, 2. Partial glosopelvectomy with bucopharyngo-mandibulectomy

The mandible resists to stress forces during mastication. This is the reason why surgeons must use a multiangle mandibulotomy incision line. Further more, the stronger bone formation is better when there is a bigger surface for ostoesynthesis. Blood supply of the mandible must be preserved if the resection margins do not involve the vessels. Radionecrosis of the mandible can occur in particular cases in which the resection is extensive. (8)

Oncology principles tell us to operate on the tumor and the lymph node metastasis in the same surgical intervention son the neck dissection needs to include lymph node levels I to IV (9). Depending on the imaging studies the surgeon can determine wether there is a need for a functional neck dissection or a radical one is needed. The preservation of the sternocleidomastoid muscle, if possible, may facilitate the reconstruction of the floor of the mouth and the closing of the oro-tegumentary fistula that resides from the resection. Radical neck dissection with the resection of the spinal nerve may lead to impairment in the mobility of the shoulder which can be translated in a drop of the quality of life of the patient. The spinal nerve needs to be preserved if the lymph node metastasis does not involve it. Surgical treatment includes a tracheostomy performed preferably at the begining of the intervention due to the fact that intubation is difficult when the floor of the mouth and the root of the tongue are involved in the tumor process. When passing through the bucopharynx narrowing with the intubation tube there is a possibility to tear pieces of vegetant tumors and place them inside the lungs. Distant site metastasis can occur by this procedure. The safer method of placing the patient under general anesthesia is by local anesthesia tracheotomy, although this is an inconvenience for the patient. The tracheostomy will be kept until the pacient will have finished the radiotherapy.

Nutritional status needs to be assessed prior to the start of the oncology treatment and needs to be tailored according to the patient's nutritional needs. We use the NRS 2002 score to identify patients who need nutritional therapy. Enteral feeding via oral intake is prefered if the normal oral feeding is to be restored after 3 weeks. If the time interval for enteral feeding exceds 3 weeks a percutaneous endoscopic gastrostomy is prefered.

\section{DISCUSSIONS}

General opinion on the approach of the T4 malignant tumors of the oral cavity and pharynx is that the best way to ensure radical resection is to use a transmandibular approach. This type of interventions need to be performed in excelence medical centers where surgery teams have a routine and are specialised in head and neck cancer surgery.

This type of cancer leads to functional and aesthetic impairment therefor patients need to be very well informed about the entire oncology management of individual cases and the patient needs to express the written informed consent on the entire plan of therapy.

Interdisciplinary examination and consults are mandatory for the tailored individual therapy management plan. The oncology team needs to be lead by the surgeon in locally advanced cases because of the main aspect of the oncology therapy plan which is surgery. 
The use of the transmandibular approach in managing cases of locally advanced tumors of the oral cavity and pharynx has some complications that can be avoided if a propper surgical technique is used. The instruments needed to perform such an intervention are either cold or power aided. The ude of power aided tools increase precision and shorten the time needed for osteotomy and osteosynthesis.

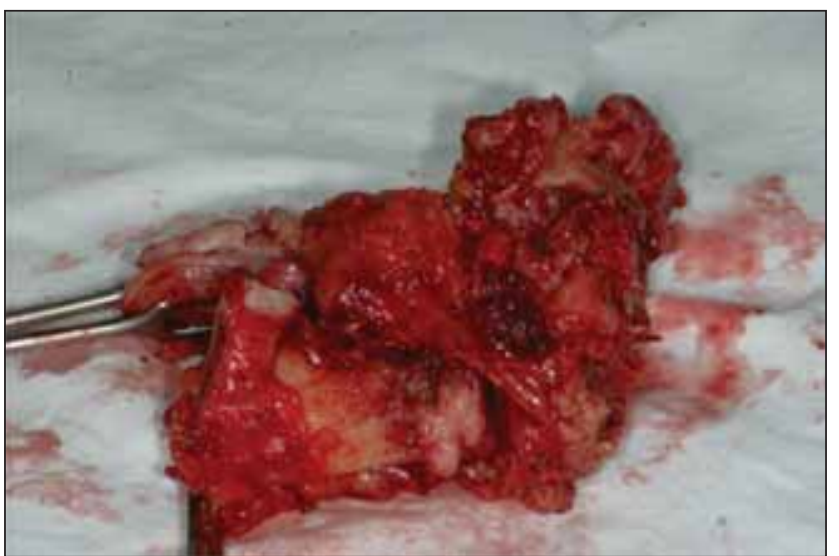

FIGURE 3. Median approach for hemiglosopelvectomy

Transmandibular technique leads to a good quality of life for the patients despite the complications of the surgery which can vary in severity from infection, bleeding and teeth denervation to lack of bone osteosynthesis, loss of teeth, abnormal movement of the mandible, lack of substance in the floor of the mouth. Wound infection is the most encoutered complication with $13,44 \%$ in our study, close to the complication rate reported in the world specialty literature of $20 \%$. (10) Complications are di- rectly related to the site of the tumor, size of the tumor, nutritional and immune status of the patient, surgical technique and postoperative care.

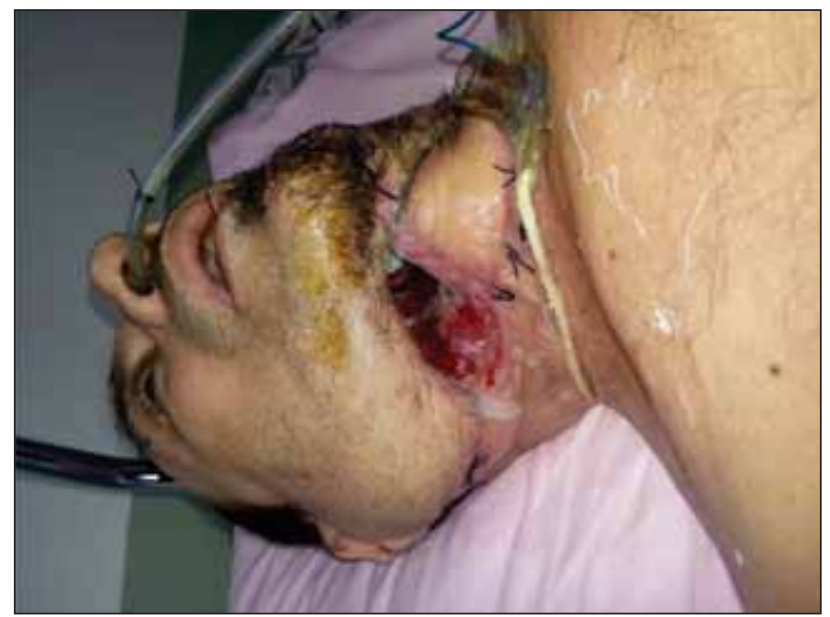

FIGURE 4. Large submanbibular fistulae after lateral approach with mandibulotomy for bucopharyngectomy

The type of mandibulotomy performed will be chosen by the surgeon accordingly to the site of the tumor and for the best access to resect the tumor. Transmandibular approach for oral cavity and pharynx malignant tumors is a safe procedure with a low rate of complications and the best oncology outcome.

\section{Acknowledge}

This paper is o part of the research project no 28483/30X2012: „Impact of biofilm development in pharyngoesophageal reconstruction with synthetic prosthesis in surgery of neoplasms of hypopharynx and cervical esophagus".

\section{$\overline{\text { BIBLIOGRAFIE }}$}

1. El-Zohairy M.A. Straight Midline Mandibulotomy: Technique and Results of Treatment. Journal of the Egyptian Nat. Cancer Inst., Vol. 19, No. 4, December: 292-298, 2007

2. Tsung S.D., Sheng P.H., Kaiping C., Wheilin P., Hweychin Y., Nganming T. Complications of mandibulotomy, midline versus paramidline. Otolaryngol Head Neck Surg. 2003, 128: 137-41.

3. Butlin H.T. Diseases of the Tongue. Clinical Manuals for Practitioners and Students of Medicine. London Cassell. 1885, 331.

4. Trotter W. Operations for malignant diseases of the pharynx. Br J Surg. 1929; 16: 485-495.

5. Brunori A., Bruni P., Greco R., Giuffré R., Chiappetta F. Celebrating the centennial (1894-1994): Leonardo Gigli and his wire saw. J Neurosurg. 1995 Jun; 82(6):1086-90.

6. McGregor I.A., MacDonald D.G. Mandibular osteotomy in the surgical approach to oral cavity. Head Neck Surg. 1983, 5 (5): 457-462.

7. Whei-Lin P., Sheng-Po H., Yaoh-Shiang L., Kai-Ping C., Jen-Liang S. The anatomical basis for mandibulotomy: Midline Versus paramidline. Laryngoscope. 2003, 113: 377-381.

8. Krespi Y.P., Sisson G.A. Transmandibular exposure of the skull base. Am J Sur. 1984, 148: 34-38.

9. Functional implications of radical neck dissection and the impact on the quality of life for head and neck neoplasia, Journal of Medicine and Life, Vol. 5, Issue 4, 2012 